Annuaire suisse de politique de développement

15 | 1996

Annuaire Suisse - Tiers Monde 1996

\title{
Le travail des enfants : politique préventive d'une entreprise important des tapis
}

Jacques Zwahlen

\section{(2) OpenEdition}

Édition électronique

URL : http://journals.openedition.org/aspd/1528

DOI : $10.4000 /$ aspd. 1528

ISSN : 1663-9669

Éditeur

Institut de hautes études internationales et du développement

\section{Édition imprimée}

Date de publication : 1 mars 1996

Pagination : 265-270

ISSN : 1660-5934

\section{Référence électronique}

Jacques Zwahlen, "Le travail des enfants : politique préventive d'une entreprise important des tapis », Annuaire suisse de politique de développement [En ligne], 15 | 1996, mis en ligne le 16 mai 2013, consulté le 08 septembre 2020. URL : http://journals.openedition.org/aspd/1528 ; DOI : https:// doi.org/10.4000/aspd. 1528 


\section{Le travail des enfants: politique préventive d'une entreprise important des tapis}

\section{Jacques Zwahlen}

La détermination d'un corps de règles et de comportements destiné à éviter dans ses relations d'affaires toute implication dans des réseaux recourant au travail forcé des enfants est récente pour l'entreprise Veillon'. Elle a coïncidé avec l'élargissement l'année dernière de l'assortiment principal à des articles textiles n'appartenant pas au secteur de l'habillement, coeur traditionnel des lignes de produits présentées dans le catalogue de la société.

Parmi les nouveaux articles figurait une collection de tapis. En préparant cette dernière, l'entreprise n'avait pas pris de précautions particulières en ce qui concerne les conditions de travail pratiquées dans cette industrie. C'est la mésaventure d'une entreprise ${ }^{2}$, répercutée dans la presse au moment même où était lancée la collection, qui a alerté Veillon du risque important encouru par les distributeurs occidentaux de se trouver pris sans le savoir dans des filières exploitant intensivement et systématiquement une main-d'oeuvre enfantine âgée de moins de dix ans.

Le tapis a ceci de spécifique par rapport aux autres produits textiles que son degré de qualité est proportionnel à la finesse des doigts qui le fabriquent. Ainsi, les mains des enfants en bas âge conviennent particulièrement à la bonne facture des noeuds petits et serrés qui procurent le plaisir esthétique de l'acheteur potentiel.

1. Veillon SA est une entreprise suisse de VPC (vente par correspondance) spécialisée dans la mode et distribuant principalement des articles textiles (habillement, tapis, rideaux, literie). Le chiffre d'affaires de 1994 s'est élevé à 185 millions de francs.

2. Des journalistes suédois, en remontant la filière d'un fournisseur, ont découvert et révélé que des tapis vendus par IKEA étaient fabriqués par des petits enfants. 
Le cas de ce produit déborde largement la question du travail des jeunes adolescents dans les pays en voie d'industrialisation. II ne peut pas non plus être assimilé à une forme de travail intégrateur tel que le pratiquent certaines économies traditionnelles tribales ou familiales. II touche des filles et des garçons de 4 à 10 ans que l'on prive de tout avenir en leur interdisant l'accès à l'école, en en réduisant leurs liens affectifs avec la famille et en les épuisant prématurément à raison de 10 à 12 heures de labeur journalier. Afin de les rendre productifs, les patrons appliquent des méthodes violentes, en les battant ou en les enchaînant à leur poste de travail. Enfin, comme les mesures de sécurité sont inexistantes, les enfants se blessent fréquemment avec les couteaux qu'ils utilisent continuellement pour couper les fils de chaque noeud qu'ils viennent de nouer.

Après avoir pris conscience de cette dramatique réalité, l'entreprise a décidé de se doter d'une politique claire et ferme afin de pouvoir assurer la cohérence de ses comportements et relations d'affaires avec ses valeurs, sa culture, de même que ses intérêts commerciaux bien compris.

Outre le fait que Veillon est une société désireuse de fonder son développement sur des valeurs respectueuses de la personne humaine ${ }^{3}$, elle est aussi convaincue que la garantie d'une "compatibilité sociale" sera toujours plus exigée par les consommateurs, cela d'autant plus si le produit comporte des risques spécifiques et connus par un large public ${ }^{4}$.

\section{Prise de conscience}

Pour assurer une base solide à cette démarche, il est apparu indispensable de faire appel à une compétence extérieure, experte dans le domaine. Contact a immédiatement été pris avec Carlos Bauverd, alors porte-parole du BIT et spécialiste des questions liées à l'esclavage. Une séance de sensibilisation au problème du travail forcé fut organisée à l'attention des collaborateurs concernés. L'objectif était d'abord de prendre conscience de l'envergure de ce fléau et du fait qu'il constitue aujourd'hui une vraie menace à l'équilibre économique mondial. A travers l'exposé de l'expert du BIT, appuyé par un reportage vidéo, chaque acheteur Veillon responsable d'une section de l'assortiment a ensuite pu percevoir la mesure du risque encouru dans son secteur.

La discussion qui s'en est suivie a montré à quel point chacun, à ce moment-là, sous-estimait encore ce problème et se trouvait profondément choqué par l'ampleur qu'il avait pris durant la dernière décennie. D'après les informations fournies par $\mathrm{C}$. Bauverd, sur les 42 pays avec lesquels Veillon entretient des relations d'affaires, seuls 19 pays ne présentent pas de risques ${ }^{5}$ du point de vue de l'esclavage de main-d'oeuvre adulte ou enfantine.

3. En 1993, une Charte a été adoptée par l'ensemble des 600 collaborateurs afin de formuler les valeurs et comportements essentiels de l'entreprise.

4. Le tapis remplit typiquement aujourd'hui ces deux conditions.

5. Cette estimation est faite en considération des critères définis par le BIT. 
En ce qui concerne le travail forcé des enfants, la région la plus problématique reste le sous-continent indien où de larges secteurs de l'économie utilisent des jeunes de moins de douze ans. L'expert du BIT a néanmoins clairement rappelé que le risque ne se limitait pas à cette zone du globe. II convenait donc de demeurer vigilant dans l'ensemble des relations avec les fournisseurs.

\section{Élaboration de règles et prise de mesures concrètes}

Forts de ce constat, les responsables de l'entreprise ont élaboré des règles à respecter dans les relations avec les fabricants. Le premier principe tend à favoriser au maximum la collaboration à long terme avec les fournisseurs afin de permettre le développement d'un véritable pacte de confiance et de transparence réciproques. Cette exigence s'accompagne naturellement de celle de contacts directs, sur place, supprimant autant que possible toute forme d'intermédiaires. Outre son avantage éminemment économique, cette solution permet d'instaurer, pratiquement sans effet de force, une surveillance systématique des conditions de production des articles commandés. A l'origine, cette surveillance visait essentiellement le contrôle de la qualité des produits. Elle s'étend désormais aux conditions de travail. Celles-ci ne sont bien sûr pas examinées du point de vue des normes en vigueur en Europe. La situation industrielle des pays riches ne peut être calquée telle quelle sur les pays en voie de développement. A l'inverse, un relativisme socioculturel absolu s'avère dangereux dès l'instant où les conditions de travail se retournent contre les intérêts mêmes des économies locales des pays pauvres, en y cultivant une logique, non plus de production, mais littéralement de destruction des ressources indigènes. Tel est le cas du travail forcé des enfants. Celui-ci use à très court terme les forces vives de tout jeunes travailleurs, réduisant au chômage les plus âgés et tarissant la source naturelle du travail en interdisant le renouvellement de générations formées, aptes à la production de vraies richesses pour le développement durable de la société.

Les critères que Veillon s'est donnés pour définir le travail forcé sont les suivants:

a) Impossibilité pour la personne de quitter ou de changer de travail.

b) Impossibilité de sortir de l'entreprise, de jour comme de nuit.

c) Recours à un système punitif dégradant tel que l'administration de châtiments corporels.

d) Absence ou quasi-absence de rémunération ${ }^{6}$.

e) Impossibilité d'acquérir une éducation de base.

6. Sont aussi comprises ici toutes les formes d'esclavage pour dettes. 
En outre, sur le terrain, la surveillance systématique que Veillon exerce se décline en exigences concrètes. Dans tous les cas, les responsables doivent pouvoir obtenir les adresses des fabriques et visiter les sites de production à leur convenance. Lorsque les produits sont particulièrement sensibles, ces exigences sont renforcées sur les points suivants:

a) Pouvoir visiter les usines sans préavis et dans l'heure qui suit.

b) Pouvoir parler librement aux personnes qui travaillent dans les ateliers, particulièrement à celles d'allure jeune, et s'assurer qu'elles puissent répondre relativement ouvertement et normalement.

c) Contrôler les mains des ouvriers afin de s'assurer qu'elles ne sont pas blessées, couvertes de plaies ou de cicatrices. A travers cela, obtenir la garantie de l'existence d'une prise en charge médicale en cas d'accident.

d) S'assurer personnellement que les travailleurs quittent le site de production en fin de journée, que l'usine n'est pas un lieu de détention et qu'elle ferme la nuit.

e) Lorsque cela est possible, faire examiner la marchandise par un expert apte à déceler dans la texture du produit si celui-ci a été réalisé par des mains enfantines.

f) Pour les jeunes adolescents (10-12 ans) au travail, s'assurer qu'ils reçoivent une éducation de base.

Dans le tapis, l'application stricte de ces règles a abouti à des corrections significatives et rapides dans la politique d'assortiment. Le responsable concerné a interrompu la collaboration avec deux grossistes incapables de fournir les adresses de ses fabriques en Inde et au Pakistan. De nouvelles sources d'approvisionnement ont été prospectées en Afrique du Nord. Un nouveau fournisseur a été trouvé au Maroc, prêt à jouer le jeu de la transparence. Des contrôles immédiats sans préavis ont pu être effectués dans les lieux de production. L'acheteur a pu entrer en contact direct avec les ouvriers et s'assurer de leurs conditions de travail acceptables.

D'une manière générale, chaque responsable d'achat répond désormais de la qualité de ses produits du point de vue des conditions de travail pratiquées. L'entreprise reste enfin en contact avec Carlos Bauverd actuellement en charge de ce dossier pour l'Association François-Xavier Bagnoud, afin de pouvoir, en cas de besoin et en tout temps, être mise en relation avec des réseaux locaux de surveillance tels que ceux développés en Inde et au Pakistan par le Front de libération du travail forcé ou par d'autres ONG et partenaires sociaux locaux.

Ces mesures simples et concrètes n'exigent pratiquement pas d'investissement financier supplémentaire. Par contre, elles engagent les responsables concernés qui doivent s'impliquer avec motivation et doigté dans des discussions et des échanges délicats avec des fournisseurs qui ne parlent pas la même langue, ni ne partagent les mêmes valeurs. Elles obligent l'acheteur à se déplacer, à prospecter systématiquement, et à ne pas se contenter de filières toutes balisées par des intermédiaires dont l'éthique commerciale n'est pas contrôlable. 


\section{Soutien au développement}

Les mesures de prévention et de corrections telles que celles prises par Veillon dans le domaine du tapis ne peuvent, à elles seules, suffire à contrecarrer la logique du travail forcé des enfants, et cela même si elles devaient être adoptées systématiquement par l'ensemble des distributeurs occidentaux. Certes, les commandes passées par les grands groupes américains ou européens constituent un formidable moyen de pression auprès des fournisseurs susceptibles de recourir à l'esclavage. Ainsi le boycott de réseaux malfaisants peut forcer l'industrie à la reconversion et à l'assainissement des conditions de travail pratiquées dans les fabriques. II serait cependant utopique, voire même cynique, d'imaginer que cette politique de prévention, aussi juste et nécessaire fût-elle, puisse être suffisante pour assurer un redéploiement des industries incriminées satisfaisant du point de vue du développement. Car le désinvestissement pur et simple fait courir le risque d'une destruction de l'économie locale sans solution de remplacement. Les enfants sont alors complètement livrés à eux-mêmes, abandonnés à un environnement souvent encore très hostile.

L'exigence de cohérence, tant économique qu'éthique, oblige l'entreprise occidentale à contribuer aux efforts consentis en matière d'éducation et de formation professionnelles par des entrepreneurs locaux soucieux du développement à long terme de leur société. Ces personnes existent mais, sans assistance, n'ont souvent pas les moyens de concrétiser leurs intentions. Des organismes occidentaux l'ont aujourd'hui compris qui proposent aux maisons de distribution de participer, même modestement et de manière très ciblée, à la construction d'un mode de développement économique durable dans les pays fournisseurs. Le label Fair and Care indique par exemple le prélèvement d'un pourcentage sur les produits importés pour le financement d'écoles ou de centres d'apprentissage. La rug marque assure la qualité sociale des articles vendus. Quant au programme Ecotex, moyennant une cotisation de DM 30'000 par année, il met en contact le distributeur avec des fabriques qui dispensent pendant la moitié du temps de travail une éducation scolaire aux jeunes ouvriers. Enfin, à travers la fondation STEP ${ }^{7}$, les organismes suisses d'entraide mettent actuellement au point un programme permettant à des entreprises spécialisées dans la vente de tapis de contribuer à des programmes de développement. STEP propose aussi un code de bonne conduite et une surveillance neutre auprès des fabricants. Ces initiatives sont certes encore à leur début, mais elles méritent toute l'attention des entreprises désireuses d'inverser le cours des choses dans le domaine du travail forcé.

En guise de conclusion, on peut affirmer qu'une approche qui cherche à concilier les exigences souvent contradictoires de l'éthique et du commerce passe, au-delà d'une nécessaire et douloureuse prise de conscience, par une pratique à long terme. Celle-ci doit chercher à faire converger des énergies et

7. Stiftung für gerechte Bedingungen in Teppich-Herstellung und Handel. 
des bonnes volontés qui commencent à se manifester et à se concrétiser aujourd'hui, un peu partout dans le monde, pour faire refluer l'esclavage, cette régression moderne dont personne ne saurait vraiment jamais tirer parti. Soulignons que le succès de cette entreprise passe d'abord, en amont, par l'engagement sur le terrain d'acheteurs professionnels, hommes ou femmes, que rien ne destine ni ne prépare a priori à mener la bataille contre le mal-développement. Elle doit ensuite, en aval, trouver son prolongement dans une communication appropriée, simple, claire et facilement reconnaissable par les consommateurs. Ne perdons pas de vue que l'issue finale de la guerre contre l'esclavage se joue dans les innombrables actes d'achat accomplis quotidiennement par Madame et Monsieur tout-le-monde dans les magasins comme au travers des catalogues. Ici le client n'est plus seulement roi. II devient, suivant qu'il se laisse guider par son unique bon plaisir, son sens civique, sa capacité d'émotion ou encore sa faculté d'oubli, soit bourreau, soit civilisateur. A chacun de choisir.

N'observe-t-on pas souvent que le succès d'une collection de mode ne tient qu'à un fil? Puisse à l'avenir ce fil dépendre aussi de l'éducation et de l'épanouissement de celles et ceux qui, encore enfants, contribuent à la performance et à la compétitivité de l'économie contemporaine. 